\title{
The Experience of Child Neuropsychiatry Residents who Volunteered in Italian COVID-19-Designated Hospitals
}

\author{
Stefano Parravicini ${ }^{1} \cdot$ Livio Provenzi $^{2}$ (D) Serena Barello ${ }^{3} \cdot$ Tiziana Nania $^{3} \cdot$ Serena Grumi $^{2} \cdot$ Elisa Rinaldi $^{2}$. \\ Simona Orcesi ${ }^{1} \cdot$ Renato Borgatti $^{1}$
}

Received: 7 October 2020 / Accepted: 22 March 2021 / Published online: 13 April 2021

(C) Academic Psychiatry 2021

\begin{abstract}
Objective During the first months of 2020, the coronavirus disease of 2019 (COVID-19) spread rapidly and soon reached a pandemic level. With the increasing number of hospitalizations, medical and nursing personnel resources were soon inadequate. As a consequence, medical volunteers became a key human resource and young medical residents in any specialty were hired on a voluntary basis to contribute to take care of patients with COVID-19. This study reports on the lived experience of residents in child neuropsychiatry who volunteered in Italian hotspot COVID-19-designated hospitals during the epidemic outbreak.

Methods A phenomenological, qualitative approach using semi-structured interviews with open-ended questions was used to obtain in-depth narratives of the experience of residents in child neuropsychiatry volunteering in North Italy COVID-19designated hospitals. All residents $(n=8)$ participated in the study. Interviews were conducted by an expert researcher trained in qualitative methods. Data analysis was performed by independent coders.

Results Five core themes could be identified from the interviews: acting as mediators on two fronts, facing the shock of COVID19 reality, capitalizing from specialty education, growing as persons and professionals, and humanizing medical care.

Conclusions This study is unique in providing an in-depth understanding of the experience of young residents in child neuropsychiatry volunteering in general hospitals during the COVID-19 pandemic in Northern Italy. The findings suggest that this experience may be highly beneficial for both the residents and the hospital quality of care. Insights for an accurate planning of residents' engagement in future healthcare emergencies are provided.
\end{abstract}

Keywords COVID-19 $\cdot$ Epidemic $\cdot$ Medical residents $\cdot$ Qualitative method $\cdot$ Volunteering

During the first months of 2020 , the coronavirus disease of 2019 (COVID-19) rapidly spread as an unprecedented pandemic [1]. The relative higher contagion and mortality rate of COVID-19 compared to other known coronaviruses have resulted in a healthcare emergency worldwide [1, 2]. Among European countries, Italy especially the Northern regions - was hit first and most dramatically [3, 4].

Livio Provenzi

livio.provenzi@mondino.it

1 Department of Brain and Behavioral Sciences, University of Pavia, Pavia, PV, Italy

2 Child Neurology and Psychiatry Unit, IRCCS Mondino Foundation, via Mondino 2, 27100 Pavia, PV, Italy

3 Department of Psychology, Università Cattolica, Milan, MI, Italy
While intensive care units rapidly exceeded their capacity potential, the resources of medical and nursing personnel soon proved to be inadequate [3] and the psychological impact on healthcare professionals emerged [5]. Consistently, the mobilization of medical volunteers soon became a key resource to face the COVID-19 outbreak [6]. Young medical residents in any specialty were hired on a voluntary basis to assist in different tasks: from helping with direct intensive care actions to providing communication support with patients' caregivers [7, 8]. To the best of our knowledge, there are no reports on the medical residents' volunteering experience in COVID-19 designated hospitals.

In this paper, we report on a qualitative phenomenological study conducted with child neuropsychiatry residents who volunteered in Northern Italy COVID-19-designated hospitals. Such an in-depth appreciation of their experience is needed to capitalize from the present pandemic and to inform adequate policy planning for the involvement of medical residents in future healthcare emergencies. 


\section{Methods}

Between April and May 2020, eight child neuropsychiatry residents of the University of Pavia (Table 1) volunteered from one to four weeks in three general hospitals and in an emergency department where patients positive for the virus were hospitalized for intensive care therapies. In June 2020, they participated to video-conference semi-structured interviews lasting 45-60 min. Open-ended questions (Table 2) were used to explore their experience volunteering in COVID-19-designated hospitals. These questions ensured key topics to be addressed, allowing however for an interactive discussion. All interviews were conducted in Italian; they were recorded and transcribed verbatim. Confidentiality was assured by using numbers instead of names and removing identifying information from the transcripts.

The analysis was conducted by three independent coders on the Italian language transcripts in order not to lose nuances of participants' original language. Translation to English language was done only for publication purposes. Researchers first read the transcriptions several times and then they highlighted significant statements. Statements sharing similar meanings were grouped together and identified with overarching labels (i.e., themes). Disagreements among coders were discussed and resolved in conference with the help of a senior author.

\section{Results}

Five core themes were identified: acting as mediators on two fronts, facing the shock of COVID-19 reality, capitalizing from specialty education, growing as persons and professionals, and humanizing medical care.

First, residents were mainly involved in indirect care actions and they had to be ready to shift between different tasks:

"I was like a wild card, providing help where and when it was necessary" (ID04)

Table 1 Sample description

\begin{tabular}{lllll}
\hline Id & Age & Sex & Specialty year & $\begin{array}{l}\text { Length of volunteering } \\
\text { experience (days) }\end{array}$ \\
\hline 01 & 27 & M & I & 20 \\
02 & 35 & F & I & 10 \\
03 & 27 & F & I & 25 \\
04 & 28 & F & II & 10 \\
05 & 28 & F & II & 15 \\
06 & 31 & F & III & 10 \\
07 & 29 & F & IV & 10 \\
08 & 30 & F & IV & 10 \\
\hline
\end{tabular}

In a setting where opportunities to communicate were partial or minimal (e.g., the ventilation devices prevented patients' communications), the residents played a key role in facilitating communications on two fronts. Indeed, they made the communications of patients with both the medical staff and their families more effective:

"I was important in helping physicians and nurses during the daily rounds with patients" (ID08); "I had to communicate with the patient's relatives about negative news, even to communicate the death of their loved one" (ID02)

Volunteer residents also took responsibility for collecting personal belongings of deceased patients and returning them to their relatives. This was another key component of facilitating communication that contributed to a virtuous circle of empathy and care.

Second, the residents abruptly faced the shock of dealing with the COVID-19 reality as they entered in the designated hospitals. The severity of patients' conditions and the impact on their families emerged abruptly in the perception of volunteers:

"Before starting this experience, the news I received on COVID was quite impalpable, and I didn't feel different from the general population just because I was a medical doctor [...] After this experience, my perception of the pandemic changed dramatically" (ID03)

All the residents reported on the differences between the usual setting in the child neuropsychiatric unit and the emergency setting of COVID-19-designated hospitals. For example, their usual experience included dealing with chronic conditions, instead of acute life-threatening ones. Moreover, findings themselves to deal with death and end of life themes were a dramatic shock:

"The most difficult part was when I had to communicate the death of this patient to his family" (ID03); "This was simply something I was unprepared to manage, both as a person and as a professional" (ID08)

The emotional shock connected with this experience was also exacerbated by the feelings of urgency and speed, which ultimately resulted in a sense of loss of control and in feelings of fear and guilt:

"There was no protocoled way to do this (...) too many factors were outside of our control" (ID02); "I was afraid of not being up to it" (ID01); "Feeling guilty for the simple fact of being afraid, as if fear were not something that should have been allowed or possible" (ID02) 
Table 2 Semi-structured interview guide

\begin{tabular}{ll}
\hline & Open question \\
\hline 1 & Can you describe the volunteering experience in COVID-19 designated hospitals in your own words? \\
$1 \mathrm{a}$ & What feelings? \\
$\mathrm{lb}$ & What did it mean for you? \\
$\mathrm{lc}$ & What positive and negative aspects? \\
2 & What is the difference between providing care due to the epidemic and working in your original clinical setting? \\
$3 \mathrm{a}$ & What challenges did you encounter? \\
$3 \mathrm{~b}$ & How did you respond? \\
$3 \mathrm{c}$ & What external support did you receive? \\
4 & What other support did you need? \\
$4 \mathrm{a}$ & Sitting here now, what are your thoughts on this experience? \\
$4 \mathrm{~b}$ & Has your professional perspective changed since directly facing this experience? \\
\hline
\end{tabular}

While they were coping with these emotions, finding the right balance between empathy and self-control rapidly became a primary achievement:

"Being able to manage emotions, without hiding them but also avoiding becoming unable to contain them during the interaction with the patients or the relatives" (ID05); "It was important for them to know that there was a physician who could understand how they felt" (ID06)

Third, residents also reported that they were able to capitalize from their specialty education. The specificity of the child neuropsychiatry specialty — which is characterized by concepts like patient-professional communication, empathy, family-centered approaches - emerged as a resource for these residents in the intensive care environment. Communication skills are a key feature of these residents' curriculum and they were especially helpful:

"We are not surgeons; we are perhaps the least medical specialty of all. Anyway, it is inherent to our profession a direct support of the relational and psychological dimensions that may enhance the quality of care with both patients and families" (ID07)

The residents were also able to capitalize on their experiences with pediatric patients to provide an empathic support to adult patients in the emergency setting:

"For example, children with autism spectrum: you almost do not heal them in the common sense of this word. And this situation was similar: we were not there to heal but to take care of patients, families and the staff" (ID07)
Fourth, this experience provided support to the residents' growth as persons and professionals. The dramatic experience in COVID-19-designated hospitals also provided the context for discovering new resources:

"I wanted to get involved, to put myself on the frontline, to find the best way to make my own contribution" (ID05); "Maybe in the future I will be better able to handle emergency situations - which are not the rule in my field, but sometimes they may happen" (ID01)

The emotional shock also catalyzed personal and emotional growth:

"I have always tended to be bashful in my personal relationship, also with the patients' parents (...) now I can feel that I am less concerned about this (...) this unlocked something emotional in me" (ID01)

Implications on professional growth were also reported, including better understanding of the need to provide clear messages and engage in empathic communications with the patients and their relatives:

"If you avoid giving the bad news because you are afraid, then you are doing your job badly" (ID04); "The difficulty I encountered in explaining to a patient with the C-PAP helmet that this was necessary for his health (...) Clear and simple communications with patients should be part of our professional toolbox" (ID08)

A final theme regarded the participants' feeling of being responsible to take care of the psychological and emotional well-being of patients, even during an acute emergency: 
"Patients simply asked us to bring emotional and physical closeness (...) So, in the end, even if she was not conscious anymore, I gently caressed her face on behalf of her daughter (...) I could feel it was something we promised to each other" (ID05)

Residents were highly sensitive to the psychological conditions of patients and provided a psychological container for emotional and affective instances that otherwise would have been partially or completely neglected:

"Once a patient entered into the COVID unit, she disappeared from the rest of the world" (ID07); "The most fragile leaves were falling, and no one was there to catch them before they reached the floor" (ID02)

The participants felt that taking care of these emotional and psychological needs was somehow complementary to the direct care provided by specialists in the intensive care units:

"It is difficult to be empathic when you have to completely focus on physical and medical care with the most severely ill patients, when you deal with survival (...) so I think I was useful, because no one was paying attention to this aspect" (ID03); "We were like salt. Salt may be not mandatory, but it gives a taste, it enriches the experience" (ID05)

\section{Discussion}

The main aim of this study was to report on child neuropsychiatry residents' experience while volunteering in COVID-19designated hospitals in Northern Italy. Five thematic clusters emerged and they are discussed here. Their analysis can hold implications on both involving residents as a key resource in the management of healthcare emergency and on developing further neuropsychiatry residents' medical education and career.

First, the residents were mainly involved in indirect care actions, supporting communications with patients, their relatives and the medical staff. They facilitated information exchanges in a setting where communications were largely at risk of being overlooked and undervalued due to the severe patients' clinical conditions and the lack of adequate human resources caused by the sudden COVID-19 outbreak [9]. The availability of personnel with a medical background that serves this interpersonal goal is of crucial importance in emergency settings as facilitating patient-professional communication may reduce the risk of errors in clinical decision-making $[10,11]$. Moreover, engaging with medical senior professionals from different backgrounds and specialties may add value to the medical education of neuropsychiatry residents by highlighting the complimentary nature of their role in a multi-professional team.
Additionally, the experience of being in contact with severely ill patients with COVID-19 was dramatic and shocking. This emotional shock was mainly evident in relation to the perceived urgency of medical care as well as their direct exposure to the patients' death. Previous literature suggested that medical staff exposed to such events may exhibit detrimental mental health effects [12] and this has also been reported for the present pandemic [13]. Although this negative psychological impact has not been explored in volunteering medical residents, the consequences of this emotional stress and burden in this population should not be underestimated. Psychological training should be included in the education program of neuropsychiatry residents in order to enhance the emotional well-being and resilience in professionals who are largely engaged in family-centered care practices.

The neuropsychiatry residents were able to benefit from skills developed during their specific education. Indeed, all the interviewees reported that their specialty medical education provided them with specific relational skills. These included the key concept of approaching the patients as human beings and the acknowledgement of the relevance of establishing a clear and empathic communication with families and relatives $[14,15]$. From this perspective, the presence of residents in child neuropsychiatry in a healthcare emergency setting can be complimentary to the critical care specialists' staff and it can promote a positive and enriching synergy. This is warranted not only to be beneficial for the career growth of the residents, but also to ensure a more person-centered care of patients in the critical care environment.

Consistently, the study participants reported that this experience was invaluable for both their personal and professional growth. They highlighted how embarking in such an emotionally intense journey helped them find new ways of managing their own emotions and feelings in their relationship with the patients. They also reported being rewarded with a strengthened feeling of confidence in their own professional identity after this experience. Finally, from the view of residents, this experience highlighted the importance of investing in emotional support for patients and their families.

The humanization of care was identified as an overarching theme in residents' narratives. The highlighted attitude toward the humanization of care is something inherent to the child neuropsychiatrist professional identity and medical education. Although the humanization of care may be perceived as nonpriority in a critical care emergency setting [16, 17], granting space and resources for the emotional well-being of patients and their families is crucial to ensure effective communication and appropriate responses to their psychological needs [18]. The engagement of volunteering residents from other specialties may be a non-negligible resource to promote a mixed-skill effective partnership during healthcare emergencies that may benefit patients, experienced professionals, and young trainees. 
This study has limitations. Our purposive sampling was limited to Italian residents in child neuropsychiatry. While this may limit the transferability of findings to other contexts, it should be highlighted that residents were volunteering during the COVID-19 outbreak in Italy, one of the most impacted regions in the Italian territory. As such, they represent a "critical case" sample. Additionally, the residents were at different years of their specialty education and they did not volunteer in the same hospital. These differences could be considered elements of variations, which have not prevented the five core themes from emerging consistently in all the interviews. We did not explore the psychological impact of volunteering on medical residents' mental health. Future studies are needed to assess the risk of burnout, posttraumatic symptoms and moral distress in this population.

This experience may offer relevant insights for future engagement of neuropsychiatry residents as a key resource in healthcare emergency management. Child neuropsychiatry residents who volunteered in COVID-19designated hospitals improved the humanization of care, facilitated communications, and allowed for a more patient-centered care. These findings should inform the planning of involvement of young volunteering medical residents during future COVID-19 waves or other healthcare emergencies. In the experiences here examined, residents asked to be assigned "on the run" and based on their own initiative. In views of other possible waves of this pandemic - or of future healthcare emergencies - these findings may contribute to plan the involvement of child neuropsychiatry residents in advance, in order to allow for their assignment to highneed contexts.

Additionally, this research suggests that opportunities for improving medical education should be offered. Volunteering in COVID-19-designated hospitals proved to be an invaluable occasion for the career development and the professional identity of these residents. Young doctors and residents who are currently developing their medical knowledge may benefit from including crossspecialty experiences that may improve their professionalism, the acquisition of broader medical skills, and the ability to engage in optimal multi-professional teams. These competencies - acquired "on the road" by child neuropsychiatry residents enrolled in this study - are crucial and may serve as a model for a more comprehensive specialty medical education during times of crisis. Expanding the medical education of young neuropsychiatrists by integrating cross-setting experiences may enrich the personal and professional growth of future professionals. As a consequence, this will make the entire field of neuropsychiatry stronger and more able to respond to large-scale healthcare emergencies.
Acknowledgments The authors are thankful for the commitment and availability of the residents both in their volunteering period and in participating in the present study. Special thanks to Melanie Iorio, Chiara Coci, and Amanda Ferrero who contributed to making the volunteer experience possible. The authors are thankful to Claudia Spinelli for supporting us in editing the final version of the manuscript.

Funding source This study was partially funded by the Italian Ministry of Health (Ricerca Corrente 2020).

\section{Declarations}

Disclosure On behalf of all authors, the corresponding author states that there is no conflict of interest.

\section{References}

1. Baud D, Qi X, Nielsen-Saines K, Musso D, Pomar L, Favre G. Real estimates of mortality following COVID-19 infection. Lancet Infect Dis. 2020;20:773.

2. Sun K, Chen J, Viboud C. Early epidemiological analysis of the coronavirus disease 2019 outbreak based on crowdsourced data: a population-level observational study. Lancet Digit Health. 2020;2: e201-8.

3. Remuzzi A, Remuzzi G. COVID-19 and Italy: what next? Lancet. 2020;395:1225-8.

4. Spina S, Marrazzo F, Migliari M, Stucchi R, Sforza A, Fumagalli R. The response of Milan's emergency medical system to the COVID19 outbreak in Italy. Lancet. 2020;395:e49-50.

5. Barello S, Palamenghi L, Graffigna G. Burnout and somatic symptoms among frontline healthcare professionals at the peak of the Italian COVID-19 pandemic. Psychiatry Res. 2020;290:113129.

6. Soled D, Goel S, Barry D, Erfani P, Joseph N, Kochis M, et al. Medical student mobilization during a crisis: lessons from a COVID-19 medical student response team. Acad Med. 2020;95: 1384-7.

7. Breazzano MP, Shen J, Abdelhakim AH, Dagi Glass L, Horowitz J, Xie SX, et al. New York City COVID-19 resident physician exposure during exponential phase of pandemic. J Clin Invest. 2020;130:4726-33.

8. Zhong BL, Luo W, Li HM, Zhang QQ, Liu XG, Li WT, et al. Knowledge, attitudes, and practices towards COVID-19 among chinese residents during the rapid rise period of the COVID-19 outbreak: a quick online cross-sectional survey. Int J Biol Sci. 2020;16:1745-52.

9. Barello S, Graffigna G. Caring for health professionals in the COVID-19 pandemic emergency: toward an "epidemic of empathy" in healthcare. Front Psychol. 2020;11:1431.

10. Woodward HI, Mytton OT, Lemer C, Yardley IE, Ellis BM, Rutter $\mathrm{PD}$, et al. What have we learned about interventions to reduce medical errors? Annu Rev Public Health. 2010;31:479-97.

11. Schiffinger M, Latzke M, Steyrer J. Two sides of the safety coin?: How patient engagement and safety climate jointly affect error occurrence in hospital units. Health Care Manag Rev. 2016;41:35667.

12. Park J-S, Lee E-H, Park N-R, Choi YH. Mental health of nurses working at a government-designated hospital during a MERS-CoV outbreak: a cross-sectional study. Arch Psychiatr Nurs. 2018;32:26.

13. Graffigna G, Barello S, Savarese M, Palamenghi L, Castellini G, Bonanomi A, et al. Measuring Italian citizens' engagement in the 
first wave of the COVID-19 pandemic containment measures. A Cross-Section Stud. 2020;15:e0238613.

14. Berman E, Heru AM. Family systems training in psychiatric residencies. Fam Process. 2005;44:321-35.

15. Rickerby ML, Roesler TA. Training child psychiatrists in familybased integrated care. Child Adolesc Psychiatr Clin N Am. 2015;24:501-15.

16. Slatore CG, Hansen L, Ganzini L, Press N, Osborne ML, Chesnutt MS, et al. Communication by nurses in the intensive care unit: qualitative analysis of domains of patient-centered care. Am J Crit Care. 2012;21:410-8.

17. Paradis E, Leslie M, Gropper MA. Interprofessional rhetoric and operational realities: an ethnographic study of rounds in four intensive care units. Adv Health Sci Educ Theory Pract. 2016;21: 735-48.

18. Kon AA, Davidson JE, Morrison W, Danis M, White DB. Shared decision-making in intensive care units: executive summary of the American college of critical care medicine and American thoracic society policy statement. Am J Respir Crit Care Med. 2016;193: 1334-6.

Publisher's Note Springer Nature remains neutral with regard to jurisdictional claims in published maps and institutional affiliations. 